\title{
Stochastic Spatiotemporal Intermittency and Noise-Induced Transition to an Absorbing Phase
}

\author{
Martin G. Zimmermann, Raúl Toral, Oreste Piro, and Maxi San Miguel \\ Instituto Mediterráneo de Estudios Avanzados (CSIC-UIB),* Campus Universitat de les Illes Balears, \\ E-07071 Palma de Mallorca, Spain
}

(Received 28 May 1999)

\begin{abstract}
We introduce a stochastic partial differential equation capable of reproducing the main features of spatiotemporal intermittency (STI). The model also displays a noise-induced transition from STI to laminarity as the noise intensity increases. Simulations and mean-field analysis are used to characterize this transition and to give a quantitative prescription of its loci in the relevant parameter space.
\end{abstract}

PACS numbers: 05.45.Jn, 05.40.Ca, 05.45.Pq, 47.27.Cn

Extended nonlinear dynamical systems often display spatiotemporal chaos (STC) [1-4]. This is a complex temporally chaotic and spatially incoherent evolution with correlations decaying in both time and space. In spite of considerable theoretical and experimental efforts devoted over the last decade to precisely define STC and its different regimes, the present status is still unsatisfactory. A useful pathway to improve our understanding of STC is the investigation of scenarios based on simple models, with few controlled ingredients, that reproduce its main characteristics. Models of this kind are instrumental in searching for generic mechanisms and universal behavior. However, it is noticeable that very few of the currently available STC scenarios belong to the framework of stochastic partial differential equations (SPDE) [5-9]. The mapping of the Kuramoto-Shivashinsky equation, paradigm of the STC regime named phase turbulence, to the Kardar-Parisi-Zhang [10] stochastic model for surface growth, is a remarkably successful exceptional example.

An important regime of STC displayed by a large variety of systems [11-14] is the so called spatiotemporal intermittency (STI). Generally speaking, this regime is a chaotic spatiotemporal evolution, the turbulent phase, irregularly and continuously interrupted by the spontaneous formation of domains with a wide range of sizes and lifetimes, where the behavior is ordered (laminar). The borders of any such laminar region evolve like fronts towards each other until the region eventually disappears in the turbulent background. STI has many features in common with some phenomena of probabilistic nature. For instance, several critical exponents at the onset of this regime often appear to belong to the universality class of directed percolation [15]. A possible connection between STI and nucleation - another process of stochastic origins - has also been pointed out [13]. However, in spite of these indications, no direct description of STI in terms of SPDEs has been put forward so far.

The purpose of this Letter is to introduce a model entirely based on a simple SPDE that displays the main features of STI, as well as to report the existence of a noise induced transition from laminarity to STI. In our model, the role of the turbulent phase is played by a strongly fluctuating noise-driven state, while the laminar phase is associated with a type of equilibrium state named absorbing. For either large values of the noise intensity or small values of the diffusion rate, the system is globally attracted to the absorbing state, but for a given finite diffusion rate, a regime of STI sets in when the noise intensity falls below a critical value. We investigate the nature of this transition both analytically, using a mean-field scheme, and numerically, by computing (a) the change of the mean velocity of the fronts between absorbing and turbulent regions, (b) the one-site probability distribution function, and (c) the average fraction of the system in the laminar phase as an order parameter.

Let us consider the following one-dimensional Itô-SPDE [16] for the evolution of a real field $u(x, t)$ [17]:

$$
u_{t}=-\frac{\partial \phi}{\partial u}+D u_{x x}+\sqrt{\epsilon} g(u) \xi .
$$

The right-hand side of this equation contains the gradient force of a potential $\phi(u)$, a diffusive term controlled by the constant $D$, and a zero-mean multiplicative Gaussian white noise $\xi(x, t)$ with $\left\langle\xi(x, t) \xi\left(x^{\prime}, t^{\prime}\right)\right\rangle=$ $2 \delta\left(x-x^{\prime}\right) \delta\left(t-t^{\prime}\right)$. The following are the two key ingredients of our model: (i) $\phi(u)$ is a nonsymmetric double well potential and (ii) the noise multiplying function $g(u)$ vanishes at the metastable minimum of $\phi(u)$.

The simple choice $g(u)=u$ [18] warrants the signpreserving evolution of $u(x, t)$ allowing us to focus on the $u(x, t) \geq 0$ case by picking only non-negative initial conditions. With this restriction in mind, we take $\phi(u)=a u^{2}-u^{4}+h u^{6}$ as a potential, with $h>0$ and $0<a<a_{M}=1 / 4 h$ a parameter range such that $\phi$ has a relative minimum at $u=0$, the absolute minimum at $u=u_{+}$and a maximum at $u=u_{-}$[19]. At one of the extremes of the $a$ range we have the "Maxwell point" $a=a_{M}$, where $\phi(0)=\phi(u+)$, whereas at the other extreme $a=0$ the equilibrium state $u=0$ changes from being metastable to unstable. In the absence of noise, fronts separating regions of $u=0$ from regions of $u=$ $u_{+}$propagate, due to diffusion, towards the metastable region at a finite speed. As a consequence, any finite region of $u=0$ surrounded by $u_{+}$will eventually shrink letting 
the system evolve to the uniform state $u_{+}$which is then a global attractor except for those initial configurations entirely laying on the small basin of $u=0$.

When $D=0$ the multiplicative noise makes of $u(x) \equiv$ 0 an absorbing state [9]: fluctuations eventually drive the system into $u(x) \equiv 0$, but neither fluctuations nor the deterministic dynamics can push the system away from this configuration. This is because the noise amplitude function $g(u)$ vanishes at the fixed point $u=0$ while field values away from it activate the fluctuations. A careful computation shows indeed that $u=0$ is technically an attracting boundary [20]. In other words, in the presence of finite noise the spatially uncoupled system always ends settling in the absorbing state since a fluctuation large enough to drive the system there eventually occurs.

From the previous analysis, we infer that noise and diffusion play opposite roles in the asymptotic space-time evolution of the field $u$. While noise nurtures the development of regions dominated by the absorbing state, diffusion favors the dominance of $u$ values fluctuating around the global minimum of $\phi(u)$. We identify the quiescent uniform absorbing state of this system with laminarity and the disordered fluctuations around the global potential minimum with turbulence. Figure 1 shows a space-time plot of a numerical solution of Eq. (1), where STI is clearly observed. Notice the occasional nucleation of segments of the laminar or absorbing state shrinking later due to the progress of the neighboring fluctuating or turbulent state. The wide range of sizes and lifetimes of laminar domains, characteristic of STI, is evident in the picture.

A similar mechanism, also involving the nucleation of a laminar state, has been invoked as responsible of STI in a deterministic reaction-diffusion system [13]. In this case, the role of noise is played by a chaotic dynamics generated via a Hopf bifurcation.

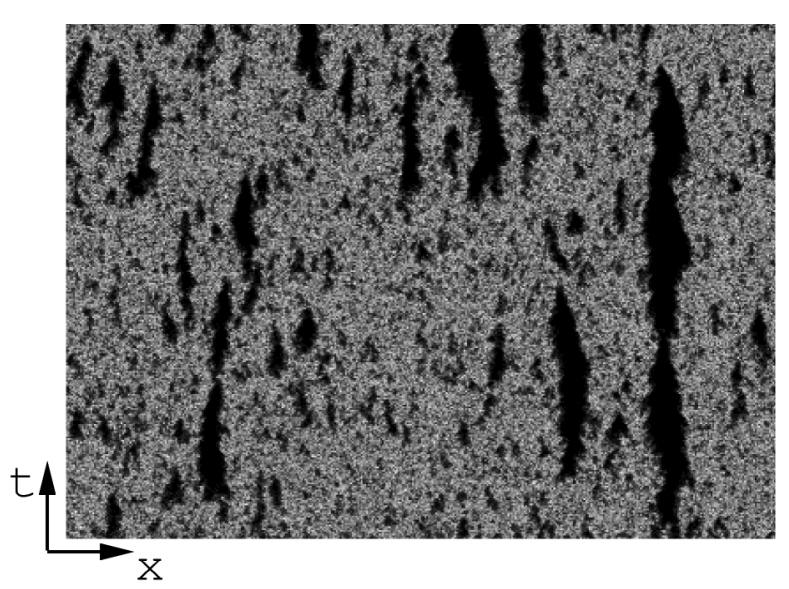

FIG. 1. Space-time evolution of $u(x, t)$ in a persistent STI regime. Black: $u=0$, grey: $u>0 . x$ and $t$ ranges are $(0,400)$ and $(0,90)$ with periodic spatial boundary positions. The initial condition is random in the interval $u_{0}(x) \in(0,2.4)$. The other parameter values are $\epsilon=0.95, a=0.5, D=2.0, h=0.22$, $\Delta x=1, \Delta t=0.001$.
In Fig. 2 we compare the evolution of fronts separating absorbing and active regions for three different noise levels but keeping $a$ and $D$ fixed. When the noise intensity $\epsilon$ is low, the fronts move in the direction expected from the deterministic limit letting the noisy phase invade the absorbing one. In this way the system asymptotically tends to a globally active phase $u_{+}$. On the other hand, for large $\epsilon$, the fronts move on average in the direction opposite to that indicated by the deterministic limit. In this case, then, the absorbing state takes over the regions in the fluctuating phase, which asymptotically tend to disappear. Finally, there is an intermediate value of $\epsilon$ for which the fronts remain at rest on average [21].

It is worth noting that this picture is consistent with the complementary results of Ref. [22]. There it is shown that the velocity of fronts connecting two equivalent potential minima is reversed if the noise working point - the value of the field where the noise amplitude function vanishes - is gradually shifted from one well to the other.

The change of the average front velocity direction as the noise intensity is varied is the key mechanism underlying a noise-induced transition from STI to an absorbing phase. To give quantitative evidence of this transition, let us consider the average spatial fraction $R$ of the system lying in the absorbing phase as an order parameter. In Fig. 3a $R$ is plotted as a function of the noise intensity $\epsilon$. Notice the transition from $R \approx 0$ (STI) to $R=1$ at about the same critical noise intensity where the front velocity reversal takes place. $R=1$ unambiguously reveals a phase uniformly dominated by the absorbing state-the laminar phase. $R \neq 1$ instead indicates that the evolution is a mixture of laminar and fluctuating domains and a closer investigation shows that it is indeed an STI regime with narrower ranges of field scales as the noise decreases. In fact, since the nucleation of absorbing domains of any size

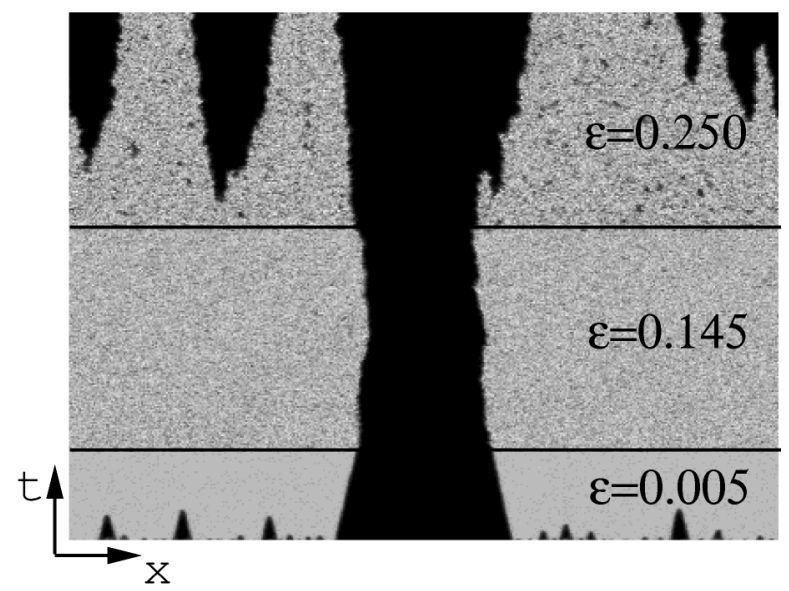

FIG. 2. Space-time plot of $u(x, t)$ starting from a random initial condition $u_{0}(x)$ of mean $u_{+}$for $x \in(0,400)$ except in the interval $x \in(150,250)$ where $u_{0}(x)=0$. The noise intensity $\epsilon$ is changed during the evolution: $\epsilon=0.005$ for $t \in(0,30)$, $\epsilon=0.145$ for $t \in(30,105)$, and $\epsilon=0.250$ for $t \in(105,180)$. Other parameters are $a=1.0, D=2.0$. 
a
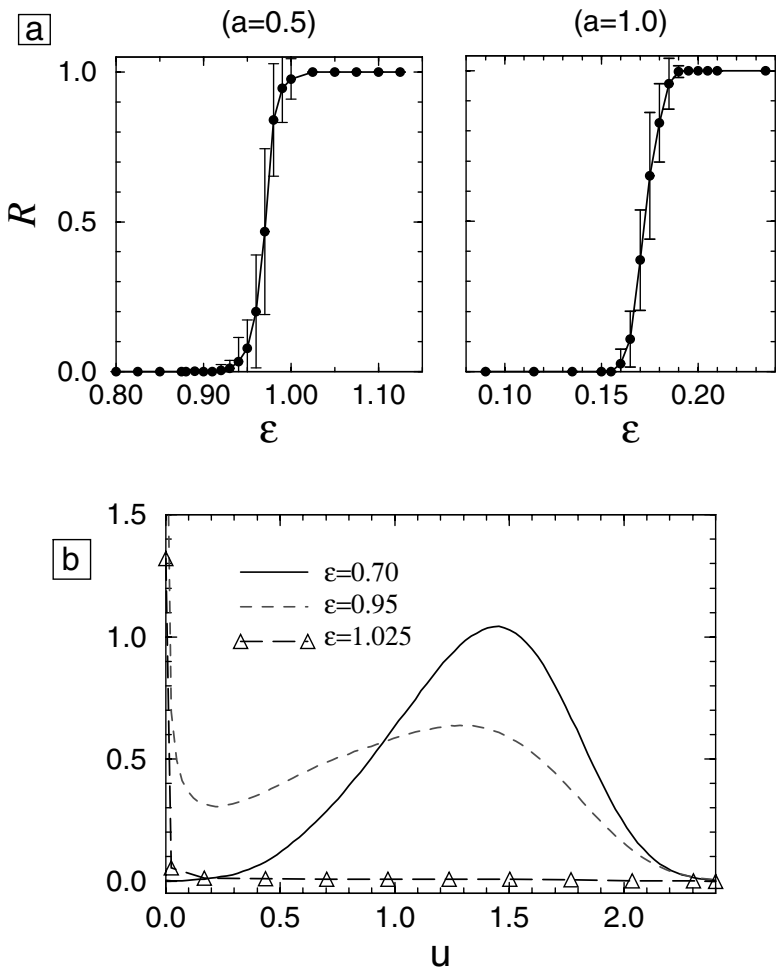

FIG. 3. (a) Fraction portion of absorbing phase $R$ as a function of $\epsilon$ and two different values of $a$. The data are the result of averaging over 40 realizations, for a time $t=900$. Other simulation parameters as in Fig. 1. (b) One-site probability distribution $P(u)$ for different values of $\epsilon$ and $a=0.5$. The ordinate axis of the pdf corresponding to $\epsilon=1.025$ is scaled down 10 times.

will occur in finite times provided that $\epsilon>0$, STI behavior is also expected in the region where $R \approx 0$, but its observation might require extremely long integration times.

Another useful measure to characterize this transition is the one-site stationary probability density function (pdf) [9], $P(u)$. In Fig. 3b we plot $P(u)$ for different values of the noise intensity. The shape of the pdf changes from being humped around the active phase $u_{+}$to being peaked at the absorbing state $u=0$. Notice that this transition occurs at the same value of $\epsilon$ where $R$ changes abruptly.

We will now examine the transition described above under the light of a Weiss-like mean-field theory specially devised to study the effects of fluctuations [23]. We first notice that by rescaling conveniently the space variable $x$ we can rewrite (1) as $u_{t}=f(u)+D_{0} u_{x x}+g(u) \xi$ with $f(u)=-\frac{\partial \phi}{\partial u}$ and $D_{0} \equiv D / \epsilon^{2}$. Next we consider the spatial discretization of the field $u_{i}=u\left(x_{i}, t\right)$, write the multivariate Fokker-Planck equation for the set of variables $\left\{u_{1}, u_{2}, \ldots\right\}$ and integrate over all the $u_{i}$ variables except one. This yields an equation for the stationary one-site pdf $P(u)$ :

$$
\partial_{u}\left\{\left(-f(u)+2 D_{0}[u-E(u)]\right) P\right\}+\partial_{u}^{2}\left(g^{2}(u) P\right)=0,
$$

where $E(u)=\int u^{\prime} P\left(u^{\prime} \mid u\right) d u^{\prime}$ is the steady-state conditional average of the field at the nearest neighbor of a site in which the field takes the value $u$. We now make the mean-field approximation $E(u) \simeq \bar{u}$, where $\bar{u}$ is the yet unknown mean value of $u$. This approach is analogous to the traditional Weiss mean-field theory of critical phenomena. Integrating (2) we obtain the steady-state pdf,

$$
P(u ; \bar{u})=\frac{1}{Z g(u)^{2}} \exp \int_{0}^{u} \frac{f(v)-2 D_{0}(v-\bar{u})}{g(v)^{2}} d v,
$$

normalized by the constant $Z$ and explicitly depending on the mean value $\bar{u}$ which must be obtained from the consistency relation

$$
\bar{u}=\int_{0}^{\infty} v P(v ; \bar{u}) d v .
$$

Equation (4) can be solved in the uncoupled limit $D_{0}=$ 0 to get $P(u) \sim u^{-2(1+a)}$ which cannot be normalized since $a>0$. Hence, $P(u)=\delta(u), \bar{u}=0$, and the system is in the absorbing phase. On the other hand, the limit $D_{0}=\infty$ can be treated using a saddle-point expansion in $D_{0}$ which leads to the equation $f(\bar{u})=0$ [24]. This equation coincides with the steady-state result of the deterministic analysis for a spatially averaged field. Such analysis predicts a transition from the absorbing state $u=0$ to the state $u=u_{+}$at the Maxwell point $a=a_{M}$. These two limiting results show that for $0<a<a_{M}$ the transition from STI to the absorbing phase must be a combined effect of fluctuations and spatial coupling. For intermediate values in the coupling $D_{0}$ we need to solve numerically the consistency relation (4). In Fig. 4 we compare the phase diagram predicted by the mean-field approach with the ones obtained from direct numerical integration of (1).

A possible variation of our model is to include a finite correlation length in the Gaussian white noise (Fig. 5). This modified model recreates the characteristic triangular structures displayed by several two-component

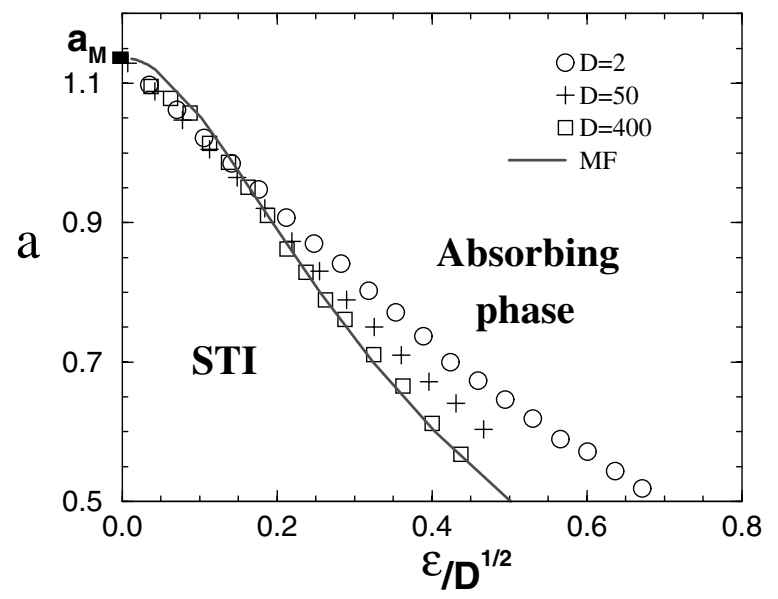

FIG. 4. Phase diagram of the noise-induced transition from STI to absorbing phase. The symbols correspond to numerical simulations of (1) for different values of $D$ and $\epsilon$. The solid line comes from the solution of the mean-field consistency (4) [25]. 


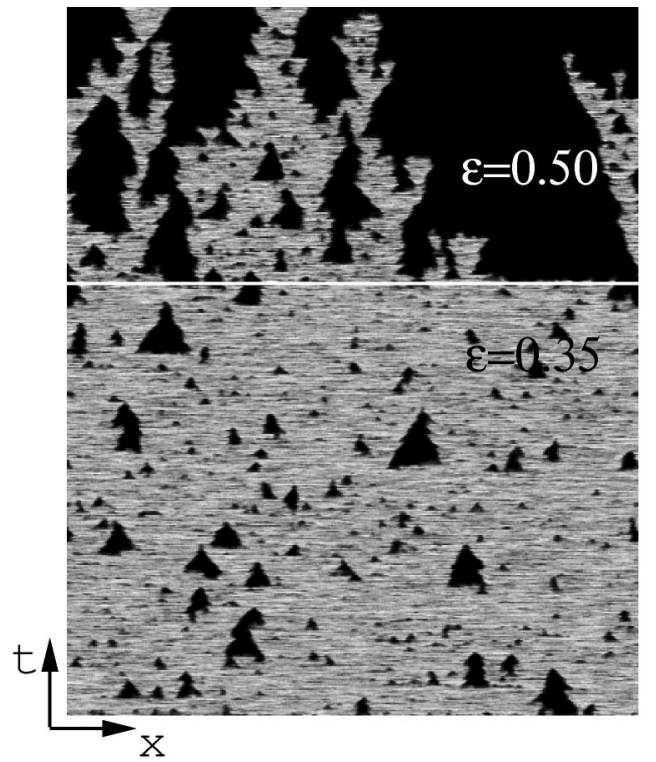

FIG. 5. Stochastic STI produced by spatially correlated noise, $\left\langle\xi(x, t) \xi\left(x^{\prime}, t^{\prime}\right)\right\rangle=\exp \left(-\frac{\left|x-x^{\prime}\right|}{\gamma}\right) \delta\left(t-t^{\prime}\right)$, with noise intensities $\epsilon=0.35$ for $t \in(0,150)$ and $\epsilon=0.50$ for $t \in(150,250) ; x \in$ $(0,400)$ and $\gamma=8.0, D=2.0$.

deterministic reaction-diffusion equations $[13,14]$. Note that in this case the transition to the laminar state occurs for nonzero velocity of the fronts, in analogy to what is observed in deterministic models [13].

In summary, we have shown that STI can be modeled by a simple SPDE. Our model clearly portrays STI as a process of stochastic nucleation of an absorbing metastable state. We also show the occurrence of a noise-induced transition where STI disappears in favor of an absorbing phase at high noise intensity or weak spatial coupling. Notice that both transitions to absorbing states $[8,9]$ and noise-induced phase transition [7,26] are known in the SPDE context. However, the noiseinduced transition shown here is unique in that it arises from the interplay between fluctuations and spatial coupling, i.e., as a genuine consequence of stochasticity in extended dynamical systems. It is also worth noting that by focusing on the spatiotemporal features of individual realizations, we are able to potentiate the applicability of the SPDE methods beyond the purely statistical realm and convert them into very useful tools to explore new generic mechanisms of spatiotemporal chaos.

We acknowledge helpful comments from J. M. Sancho and M. A. Muñoz, and financial support from DGES (Spain) Projects No. PB94-0167, and No. PB97-0141C02-01. M. G.Z. is supported by the MEC (Spain).

*Electronic address: http://www.imedea.uib.es/PhysDept [1] P.C. Hohenberg and D. Shraiman, Physica (Amsterdam) 37D, 109 (1989).
[2] M.C. Cross and P.C. Hohenberg, Science 263, 1569 (1994).

[3] T. Bohr, M.H. Jensen, G. Pladin, and A. Vulpiani, Dynamical Systems Approach to Turbulence (Cambridge University Press, Cambridge, 1998).

[4] G. Ahlers, Physica (Amsterdam) 249A, 18 (1998).

[5] J. García-Ojalvo and J.M. Sancho, Noise in Spatially Extended Systems (Springer, New York, 1999).

[6] C. Doering, Phys. Lett. A 122, 133 (1987).

[7] R. Müller, K. Lippert, A. Kühnel, and U. Behn, Phys. Rev. E 56, 2658 (1997).

[8] Y. Tu, G. Grinstein, and M. A. Muñoz, Phys. Rev. Lett. 78, 274 (1997).

[9] M. A. Muñoz, Phys. Rev. E 57, 1377-1383 (1998).

[10] V.S. L'vov and I. Procaccia, Phys. Rev. Lett. 69, 3543 (1992); P. Manneville and H. Chaté, Physica (Amsterdam) 96D, 30 (1996).

[11] H. Chaté and P. Manneville, Physica (Amsterdam) 32D, 409-422 (1988); H. Chaté, Nonlinearity 7, 185-204 (1994).

[12] A. Amengual, E. Hernández-García, R. Montagne, and M. San Miguel, Phys. Rev. Lett. 78, 4379 (1997).

[13] M. Argentina and P. Coullet, Phys. Rev. E 56, R2359 (1997); Physica (Amsterdam) 257A, 45 (1998).

[14] M. G. Zimmermann, S. O. Firle, M. A. Natiello, M. Hildebrand, M. Eiswirth, M. Bär, A. K. Bangia, and I. G. Kevrekidis, Physica (Amsterdam) 110D, 92-104 (1997).

[15] J. Rolf, T. Bohr, and M. H. Jensen, Phys. Rev. E 57, R2503 (1998).

[16] N. G. van Kampen, Stochastic Processes in Physics and Chemistry (North-Holland, Amsterdam, 1981).

[17] This SPDE is to be considered in the Itô interpretation such that the noise term does not alter either the location or the stability of the absorbing state at $u=0$.

[18] This choice makes our SPDE model in the multiplicative noise universality class [9].

[19] For the chosen potential $u_{ \pm}=\sqrt{(1 \pm \sqrt{1-3 a h}) / 3 h}$.

[20] W. Horsthemke and R. Lefever, Noise Induced Transitions (Springer-Verlag, Berlin, 1984), Chap. 5.

[21] Parameters are chosen such that nucleation events of the laminar phase occur on a much longer time scale than the one shown.

[22] L. Schimansky-Geier and Ch. Zülicke, Z. Phys. B 82, 157 (1991).

[23] C. Van den Broeck, J. M. R. Parrondo, J. Armero, and A. Hernández-Machado, Phys. Rev. E 49, 2639 (1994).

[24] Since Ref. [23] uses Stratonovich interpretation, the $D_{0} \rightarrow$ $\infty$ result there amounts to add a spurious drift term $f(u)+$ $g(u) g^{\prime}(u) / 2=0$. Our results, instead, do not follow from the effect of such a term.

[25] With $0<a<a_{M}$ and any value of $D_{0}$ the consistency equation (4) has three solutions that meet the solutions of $f(\bar{u})=0$ as $D_{0} \rightarrow \infty$. Generalizing the Maxwell construction that describes the change of relative stability of the solutions, we predict the transition at arbitrary $D_{0}$ (the solid line in Fig. 4).

[26] C. Van den Broeck, J.M. R. Parrondo, and R. Toral, Phys. Rev. Lett. 73, 3395 (1994); C. Van den Broeck, J. M. R. Parrondo, R. Toral, and R. Kawai, Phys. Rev. E 55, 4084-4094 (1997). 